\title{
Klotho Ameliorates Chemically Induced Endoplasmic Reticulum (ER) Stress Signaling
}

\author{
Srijita Banerjee ${ }^{\mathrm{a}, \mathrm{g}}$ Yanhua Zhao ${ }^{\mathrm{a}, \mathrm{g}}$ Partha S. Sarkar ${ }^{\mathrm{c}}$ Kevin P. Rosenblatt ${ }^{\dagger}$
} Ronald G. Tilton ${ }^{\mathrm{a}, \mathrm{b}, \mathrm{d}, \mathrm{e}}$ Sanjeev Choudhary ${ }^{\mathrm{a}, \mathrm{d}, \mathrm{e}}$

From Departments of aInternal Medicine, bophthalmology \& Visual Sciences, and 'Neurology, Neuroscience and Cell Biology, dSealy Center for Molecular Medicine, eInstitute for Translational Sciences; University of Texas Medical Branch, Galveston, TX; ${ }^{\mathrm{f} B r o w n}$ Foundation Institute of Molecular Medicine, University of Texas Health Science Center, Houston, $\mathrm{TX}$; ${ }^{9}$ Contributed equally to the work detailed in this manuscript

\section{Key Words}

Klotho • ER stress • Thapsigargin • Tunicamycin • Unfolded protein response

\begin{abstract}
Background: Both endoplasmic reticulum (ER) stress, a fundamental cell response associated with stress-initiated unfolded protein response (UPR), and loss of Klotho, an anti-aging hormone linked to NF-KB-induced inflammation, occur in chronic metabolic diseases such as obesity and type 2 diabetes. We investigated if the loss of Klotho is causally linked to increased ER stress. Methods: We treated human renal epithelial HK-2, alveolar epithelial A549, HEK293, and SH-SH-SY5Y neuroblastoma cells with ER stress-inducing agents, thapsigargin and/or tunicamycin. Effects of overexpression or siRNA-mediated knockdown of Klotho on UPR signaling was investigated by immunoblotting and Real-time PCR. Results: Elevated Klotho levels in HK-2 cells decreased expression of ER stress markers phosphoIRE1, XBP-1s, BiP, CHOP, pJNK, and phospho-p38, all of which were elevated in response to tunicamycin and/or thapsigargin. Similar results were observed using A549 cells for XBP-1s, $\mathrm{BiP}$, and $\mathrm{CHOP}$ in response to thapsigargin. Conversely, knockdown of Klotho in HEK 293 cells using siRNA caused further thapsigargin-induced increases in PIRE-1, XBP-1s, and BiP. Klotho overexpression in A549 cells blocked thapsigargin-induced caspase and PARP cleavage and improved cell viability. Conclusion: Our data indicate that Klotho has an important role in regulating ER stress and that loss of Klotho is causally linked to ER stress-induced apoptosis.
\end{abstract}




\section{Introduction}

Several metabolic diseases, including obesity, insulin resistance, metabolic syndrome, and diabetes, are associated with an elevated cellular and inflammatory stress [1-4]. A key player in the cellular stress response is the endoplasmic reticulum (ER), a membranous network that functions in the synthesis and processing of secretory and membrane proteins. Among the conditions that trigger ER stress are nutrient deprivation, viral infections, lipids, increased synthesis of secretory proteins, and accumulation of mutant or misfolded proteins [5-7]. Disruption of ER homeostasis due to accumulation of unfolded or misfolded proteins in the ER lumen, activates a signal transduction system called the unfolded protein response (UPR) $[8,9]$. UPR induces a number of protective pathways to restore ER homeostasis, including transient attenuation of protein translation, induction of molecular chaperones and folding enzymes, and increased degradation of misfolded proteins. Apoptotic pathways are activated to eliminate the damaged cells if these adaptive responses fail to alleviate the stress [10]. The molecular basis for the switch between prosurvival and proapoptotic UPR function is poorly understood.

To date, three ER-resident transmembrane proteins have been identified as proximal sensors of the presence of ER stress: the kinase and endoribonuclease IRE1 ( $\alpha$ and $\beta$ ), the PERK kinase, and the basic leucine-zipper transcription factor ATF6 ( $\alpha$ and $\beta$ ). IRE1 and PERK have cytoplasmic serine/threonine kinase domains. ER stress induces luminal-domaindriven homodimerization, autophosphorylation and activation [11-13]. Accumulation of unfolded proteins in the ER lumen leads to ATF6 transit to the Golgi complex, where it is processed by the proteases S1P and S2P, to produce an active transcription factor [14]. Transcription of genes downstream of ATF6 is upregulated by the translocation of the ATF6 cytoplasmic domain to the nucleus. IRE-1-dependent transcription is upregulated when the endoribonuclease domain of the activated IRE-1 molecule catalyzes the removal of a small (26-nucleotide) intron from the mRNA of the gene encoding X-box-binding protein (XBP-1). This splicing event creates a translational frame shift in XBP-1 mRNA to produce an active transcription factor $[15,16]$. PERK activation leads to the phosphorylation of the $\alpha$ subunit of the translation initiation factor eIF2, which inhibits 80 S ribosome assembly and results in a general inhibition of protein synthesis, decreasing the functional demand on ER [17].

Activation of all three components of the UPR depends on the dissociation of the proximal signaling molecule from the abundant luminal chaperone BiP/GRP78. Accumulating evidence supports the idea that BiP plays a key role in UPR signaling, not only as an ERchaperone but also as an ER-stress sensor [18-21]. BiP as a master negative regulator of the UPR provides a unifying hypothesis to account for the simultaneous activation of PERK, IRE-1 and ATF6 upon ER stress. However, why only a selective branch of the UPR appears to be more important for some developmental events or cellular functions but not the others is still not understood.

Activation of UPR can also trigger pro-apoptotic signals. Activation of the PERK-eIF2 $\alpha$ pathway leads to inhibition of translation in general, resulting in a global slowdown of cellular protein translation, preventing excessive protein load in ER-stressed cells. However, activation of this pathway enhances translation of selected small group of mRNA such as ATF-4. Consequently, ATF4 induces expression of pro-apoptotic CCAAT/enhancer-binding protein homologous protein (CHOP) (also called growth arrest and DNA damage-inducible protein 153 [GADD153]) through activation of the amino acid response element (AARE). The ATF6 pathway and the IRE-1 pathway may also induce expression of CHOP [22]. ER stress activates caspase-12 (caspase-4 in humans) localized at the ER membrane through an interaction with IRE1 and tumor necrosis factor receptor-associated factor 2 (TRAF2), inducing apoptosis. The IRE-1-TRAF2 interaction also allows for recruitment and activation of apoptosis signal-regulating kinase 1 (ASK1) and downstream c-Jun N-terminal kinase (JNK), that are involved in a variety of pro-apoptotic signaling [10]. Thus, under conditions of ER stress, there exists a delicate balance between cell survival and apoptosis, the outcome depending upon the load of stress inducer and duration of the stress. ER stress, 
inflammation and oxidative stress are significant contributors in the development of several chronic diseases, yet molecular and cellular mechanisms linking these stress pathways are poorly understood and infrequently explored in vivo.

Klotho is a single-pass transmembrane protein that is expressed in multiple tissues, but particularly in renal tubular cells [23]. Development of transgenic mice that over-express Klotho showed up to $30 \%$ longer life spans compared with wild-type mice [24, 25], suggesting that Klotho functions as an aging-suppressor gene [23, 24,26]. Klotho also confers resistance to oxidative stress primarily by activating FOXO family of transcription factors that regulate expression of superoxide dismutase (SOD2) [27]. Treatment with Klotho increased FOXO1 recruitment on the SOD2 gene promoter and increased its expression level in various cell types $[25,28,29]$. Klotho suppressed paraquat-induced lipid oxidation, suggesting a protective effect of Klotho on cellular oxidative damage. Transgenic mice overexpressing Klotho had $50 \%$ lower urinary excretion of 8-hydroxydeoxy-guanosine (8-OHdG), a biological marker of in vivo oxidative DNA damage [25]. We recently reported that Klotho modulates NF- $\kappa \mathrm{B}-$ driven inflammation, and loss of renal Klotho in $d b / d b$ mice (a type 2 diabetes model) was associated with increased production of inflammatory cytokines [30]. Here, we investigated the possibility that Klotho, an anti-aging hormone with anti-oxidant and anti-inflammatory properties, might regulate ER stress.

We induced ER stress in human embryonic kidney (HEK) 293, human kidney cortex proximal tubular (HK-2), alveolar epithelial A549, and SH-SY5Y neuroblastoma cells with two potent ER stressors, thapsigargin and tunicamycin. These agents disrupt ER homeostasis by affecting $\mathrm{ER} \mathrm{Ca}^{2+}$ pump and $\mathrm{N}$-linked glycosylation of proteins, respectively. We observed a significant reduction in ER stress responses in cells overexpressing Klotho vs controls; conversely, downregulation of Klotho in cultured cells (by siRNA) resulted in an increased ER stress and activation of UPR signaling. These results provide strong evidence for a potentially novel ER stress regulation by Klotho.

\section{Materials and Methods}

\section{Cell culture}

HEK 293 cells were cultured in DMEM media supplemented with $10 \%$ FBS, 50 units $/ \mathrm{ml}$ penicillin and $50 \mu \mathrm{g} / \mathrm{ml}$ streptomycin in an atmosphere of $5 \% \mathrm{CO}_{2}$, at $37^{\circ} \mathrm{C}$. A retrovirus cloning system was used to deliver the mouse Klotho gene into human kidney cortex proximal tubular cells (HK-2) and SH-SY5Y neuroblastoma cells to generate Klotho stable cell lines, HK-2/KL and SH-SY5Y/KL respectively. Both SH-SY5Y/KL and HK-2/KL cells as well as their respective control cell lines were grown and maintained in DMEM/Ham's F12 (50/50) supplemented with $10 \%$ FBS, 50 units/mL penicillin and $50 \mu \mathrm{g} / \mathrm{mL}$ streptomycin, epidermal growth factor $(2.5 \mathrm{ng} / \mathrm{mL})$, and bovine pituitary extract $(1.5 \mu \mathrm{g} / \mathrm{mL})$ in a humidified atmosphere of $5 \%$ $\mathrm{CO}_{2}$. Both growth factors were removed from the culture media 48 hours prior to experiments with stress inducers. Cells were treated with tunicamycin $(5 \mu \mathrm{g} / \mathrm{mL})$ and thapsigargin $(500 \mathrm{nM})$ for indicated times to induce ER stress.

\section{Retroviral infection and preparation of stable cell lines}

Mouse transmembrane form of Klotho containing a C-terminus Flag tag (pEFmKLcFT) was cloned into the retroviral expression vector pCX4-puro. Retrovirus stocks produced in Bosc23 cells were co-transfected with pCX4-pur-mKL-flag plasmid and amphotrophic packaging plasmid pCL-10A1. A549, HK-2, and SHSY5Y cells were infected and selected for puromycin resistance ( 2.0 and $0.5 \mu \mathrm{g} / \mathrm{ml}$, respectively).

\section{Transfection}

Transient transfection using lipofectamine PLUS reagent (Invitrogen) was performed according to the manufacturer's instructions. Cells were transfected with mouse Klotho plasmid or the control vector and treated with the ER stress-inducing agents $48 \mathrm{hrs}$ later. 


\section{SiRNA-mediated Klotho knockdown}

Cells were treated with $100 \mathrm{nM}$ Klotho siRNA (Dharmacon) or with $100 \mathrm{nM}$ non-specific siRNA (Dharmacon) using TransIT-siQUEST transfection reagent (Mirus) 48 hrs prior to treatment with thapsigargin (500 $\mathrm{nM})$.

\section{Preparation of cell lysates}

For whole cell lysates, culture media was removed, plates rinsed with ice-cold PBS, and cells scraped into eppendorf tubes using ice-cold PBS, and then spun at $2000 \mathrm{rpm}$ for $2 \mathrm{~min}$ at $4^{\circ} \mathrm{C}$. The supernatant was removed and 100-200 $\mu \mathrm{L} 1 \mathrm{X}$ RIPA buffer containing protease (Sigma) and phosphatase (Thermo scientific) inhibitors were added to the cell pellet for $15 \mathrm{~min}$ on ice, followed by centrifugation for $25 \mathrm{~min}$ at $4^{\circ} \mathrm{C}$ at $13,200 \mathrm{rpm}$. The supernatant was stored as whole cell lysate at $-80^{\circ} \mathrm{C}$.

\section{Western immunoblotting}

Proteins were fractionated by SDS-PAGE and transferred to nitrocellulose or polyvinylidene diflouride (PVDF) membranes (Millipore). Membranes were blocked in $5 \%$ milk or $5 \%$ BSA for 1 hr and then incubated with the indicated primary antibody at $4^{\circ} \mathrm{C}$ overnight. Membranes were washed in Tris-buffered saline, $0.1 \%$ Tween-20, and incubated with secondary antibody at $20^{\circ} \mathrm{C}$ for $1 \mathrm{hr}$. Signals were visualized on a chemiluminescence detection film using the ECL (GE Healthcare) system. $\beta$-actin was used as a loading control. Each target protein was quantified using the ImageJ software. The specific antibodies used were: phospho-IRE-1 (Abcam, ab124595), IRE-1 (Cell Signaling, catalog \# 3294), XBP-1s (Biolegend, catalog \# 647502; clone 143F), BiP (Cell Signaling, catalog \# 3177), phospho JNK (Cell Signaling, catalog \# 9251), JNK (Cell Signaling, catalog \# 9252), phospho-p38 (Cell Signaling, catalog \# 9216S; clone 28B10), p38 (Cell Signaling, catalog \# 9212), CHOP (Cell Signaling, catalog \# 2985).

\section{MTT assay for cell survival}

Klotho or empty vector expressing stable A549/HK-2 cells ( 5 - $10 \times 10^{3} /$ well) were seeded in 96 well plates overnight. Cells were further incubated for $24 \mathrm{hrs}$ with different concentrations of thapsigargin. At the end of thapsigargin treatment, cells were loaded with MTT and incubated for $3.5 \mathrm{hrs}$ at $37^{\circ} \mathrm{C}$ followed by cell lysis with MTT solvent (containing $4 \mathrm{mM} \mathrm{HCl}, 0.1 \% \mathrm{NP}-40$ ), and the absorbance read at $590 \mathrm{~nm}$.

\section{Quantitative real-time PCR}

Cellular RNA was extracted using Tri Reagent (Sigma). A total of $2 \mu$ g RNA was used for reverse transcription using the SuperScript III First-Strand Synthesis System from Invitrogen (Carlsbad, CA). A total of $2 \mu \mathrm{L}$ cDNA products was amplified in a $20-\mu \mathrm{L}$ reaction system containing $10 \mu \mathrm{L}$ iQ SYBR Green Supermix (Bio-Rad) and $400 \mathrm{nmol} / \mathrm{L}$ primer mixture. CHOP primer was purchased from SA Bioscience (Frederick, MD). All reactions were processed in a MyiQ Single Color Real-Time PCR thermocycler using a two-stepplus-melting curve program. Results were analyzed by the iQ5 program (Bio-Rad), and the data were analyzed using the $\triangle \Delta \mathrm{CT}$ method in reference to GAPDH [31].

\section{Statistical analysis}

All tabulated data are reported as mean \pm SEM, except where otherwise indicated. We tested all data for normal distribution using the Kolmogorov-Smirnov test, followed by two-way ANOVA test to evaluate overall group differences. This was followed by Tukey's post-hoc test to determine pair-wise significance if the ANOVA test indicated that a significant difference was present in the data set. In the case of only two group comparisons, a two-sample Student's t-test was performed after checking for variance distribution via Levene's test. In all cases, $p<0.05$ was considered significant.

\section{Results}

Klotho inhibits IRE-1 (ERN1) activation and subsequent splicing of XBP-1

Serine/threonine-protein kinase/endoribonuclease ERN1, a human homologue of the yeast IRE-1 gene product, is an enzyme that in humans is encoded by the ERN1 gene. In response to ER stress, N-terminal ER luminal domains of IRE-1 oligomerize to promote 
Fig. 1. Klotho inhibited ER stress-induced IRE-1 phosphorylation. HK-2 cells stably transfected with either Flag-Klotho $(\mathrm{K})$ or empty vector controls $(\mathrm{C})$ were treated with A) thapsigargin (500 nM) or B) tunicamycin $(5 \mu \mathrm{g} / \mathrm{ml})$ for the indicated times. Total and phospho-IRE1protein levels were determined by immunoblotting and representative blots are shown in A and B. $\beta$-actin served as a loading control. TG: thapsigargin, TM: tunicamycin. Quantification of three repeat TG experiments is shown in the lower panel in Fig. 1A. Results are plotted as mean \pm SEM. Significantly different from control (black bars) baseline: ${ }^{*} \mathrm{p}<0.01,{ }^{* *} \mathrm{p}<0.001$; significantly different from Klotho-treated (gray bars) baseline: \# p<0.01; significantly different from respective control at 6 hours: $\dagger \mathrm{p}<0.01$. TM experiment performed once to confirm TG results.

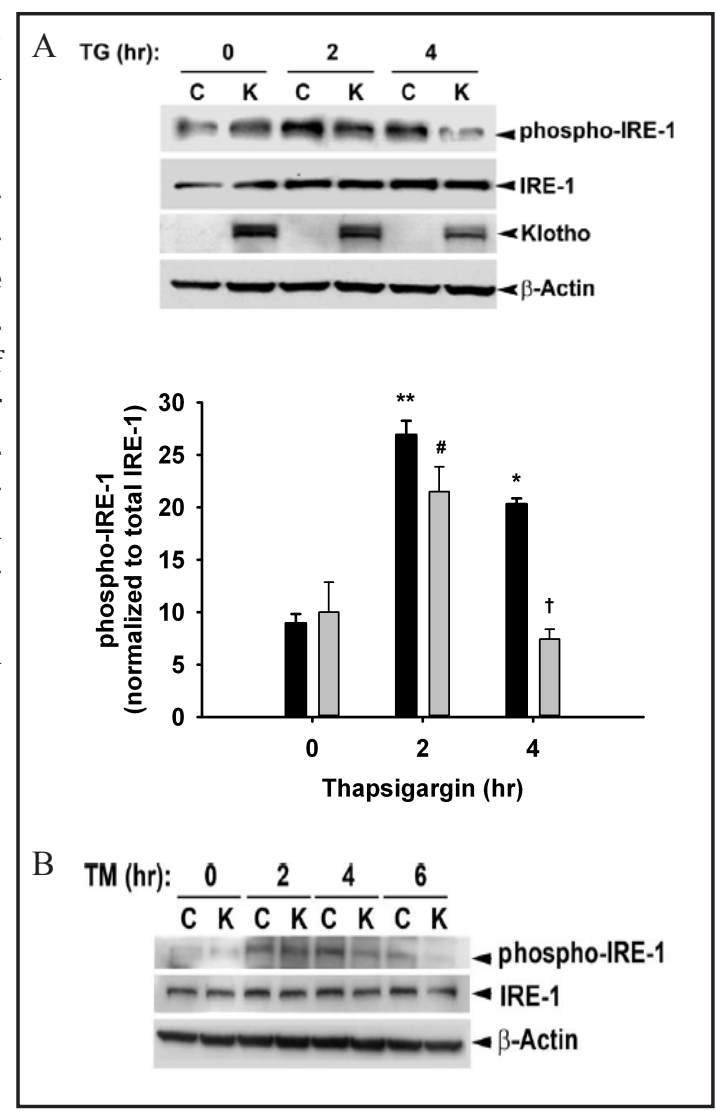

autophosphorylation by its cytoplasmic kinase domain, leading to activation of the endoribonuclease function present in the cytoplasmic domain that splices XBP-1 mRNA, generating an active XBP-1s transcriptional activator [32, 33]. Spliced XBP-1 mRNA encodes a transcription activator that drives transcription of ER chaperone genes, whose products directly participate in ER protein folding [34].

We treated HK-2 control and Klotho overexpressing cells with thapsigargin (TG; 500 $\mathrm{nM}$ ) or tunicamycin (TM; $5 \mu \mathrm{g} / \mathrm{mL}$; Figs. $1 \mathrm{~A}$ and 1B, respectively) for 2,4 and 6 hrs (TM only). Increased phosphorylation was observed following treatment with ER stress inducers within $2 \mathrm{hrs}$, and was reduced in Klotho over expressing cells by $4 \mathrm{hrs}$ in both TG- and TM-treated cells. To determine the activation status of IRE- 1 after ER stress, we examined XBP-1 splicing by Western blot. In agreement with our previous finding, we found a diminution of XBP-1s in Klotho-overexpressing cells treated with TG (Fig. 2A) as early as $2 \mathrm{hrs}$ after initiating stress treatment, becoming highly significant by 6 hours. Comparable results were observed using TM (Fig. 2B). We made similar observations in HEK293 cells transiently transfected with Klotho (data not shown). These results indicate that Klotho inhibits IRE-1 signaling under conditions of ER stress. To assess whether the UPR branch elicited by PERK is also affected by Klotho, we assayed phosphorylation of PERK. We were not able to detect PERK activation using tunicamycin or thapsigargin in the given cell types (data not shown). We repeated these experiments using SH-SY5Y neural cells that previously have been shown to activate this signaling pathway [35]. For this, the control or Klotho overexpressing stably transfected SH-SY5Y cells were treated with thapsigargin for 0 and $4 \mathrm{hr}$ followed by immunoblotting for phospho-PERK and phospho-elF2 $\alpha$. A $60 \%$ induction in PERK phosphorylation was observed in TG-treated control cells, whereas no induction was observed in TG-treated, Klotho-overexpressing cells (Fig. 2C). A more robust elF $2 \alpha$ phosphorylation in TG-treated SHSY5Y cells was completely blocked by Klotho overexpression (Fig. 2D). A significant decline in PERK and elF $2 \alpha$ phosphorylation in these cells vs control cells suggests an inhibitory role of Klotho in TG-induced PERK pathway activation. 


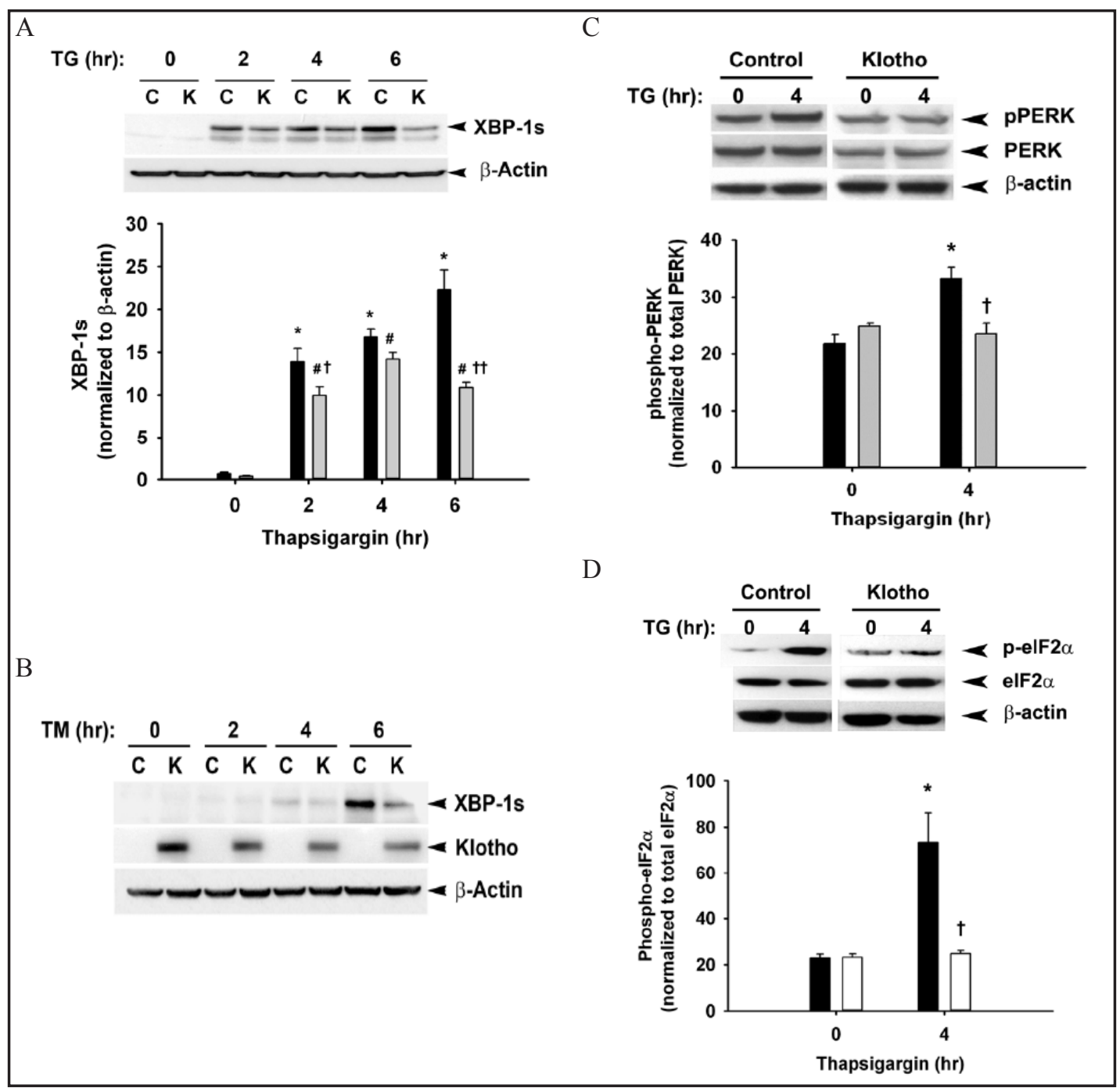

Fig. 2. Klotho reduced ER stress-induced XBP-1 splicing. HK-2 cells stably overexpressing Klotho (K) or a control vector (C) were subjected to A) thapsigargin (500 $\mathrm{nM}$ ) or B) tunicamycin $(5 \mu \mathrm{g} / \mathrm{ml})$ for indicated times. A representative Western blot shows spliced XBP-1 (XBP-1s). Expression of recombinant Flag-Klotho was determined by probing the membrane with anti-Flag (Fig. 2B). Quantification of three repeat TG experiments is shown in the lower panel in Fig. 2A. Results are plotted as mean \pm SEM. Significantly different from control (black bars) baseline: ${ }^{*} \mathrm{p}<0.001$; significantly different from Klotho-treated (gray bars) baseline: $\# \mathrm{p}<0.001$; significantly different from respective control at 2 hours: $\uparrow \mathrm{p}<0.05$; significantly different from respective control at 6 hours: $\dagger \dagger \mathrm{p}<0.001$. TM experiment performed once to confirm TG results. Control SH-SY5Y and SY5Y/KL cells were treated with thapsigargin for indicated time and immunoblotted for C) phospho-PERP and D) phospho-elF2 $\alpha$. Both figures show a representative Western blot. Immunoblots were probed with $\beta$-actin for loading control.Quantification of three repeat TG experiments is shown in the lower panel of each Figure. Results are plotted as mean \pm SEM. Significantly different from control (black bars) baseline: ${ }^{*} \mathrm{p}<0.001$; significantly different from control at 4 hours: $\dagger \mathrm{p}<0.01$. Klotho-expressing samples (gray bars). TG: thapsigargin, TM: tunicamycin.

\section{Klotho decreases BiP levels by inhibiting ER stress}

The ER luminal protein BiP is believed to serve as an ER stress sensor, triggering UPR. Under ER stress, the translation efficiency of BiP transcripts is enhanced, allowing cells to produce more protein. Western blot analysis indicated that BiP/GRP78 levels were less in Klotho-overexpressing cells 6 hrs after TG and TM stimulation (Figs. 3A and B). A beneficial 


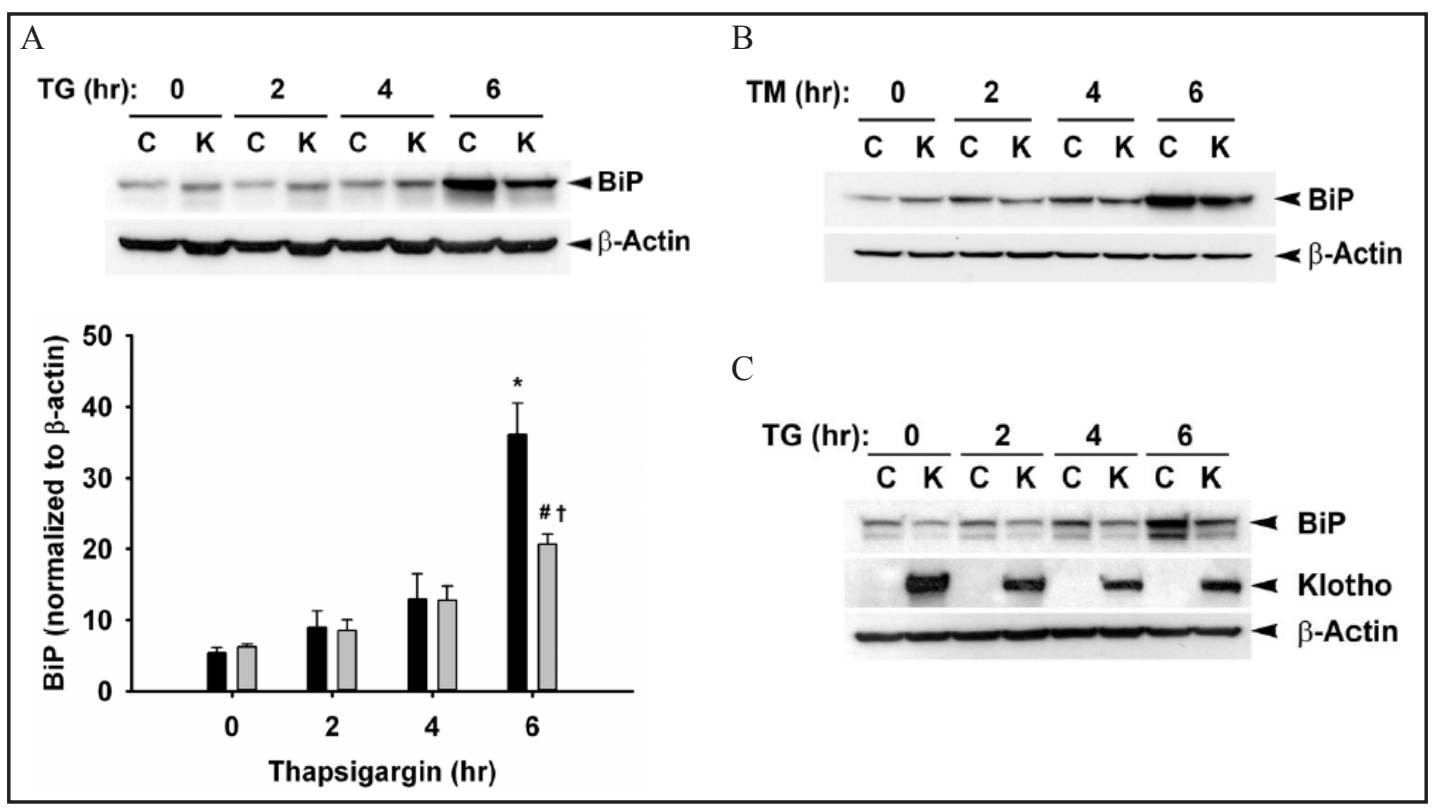

Fig. 3. Klotho significantly reduced BiP induction by ER inducers. HK-2 cells stably overexpressing Klotho were treated with A) thapsigargin $(500 \mathrm{nM})$ or B) tunicamycin $(5 \mu \mathrm{g} / \mathrm{ml})$ for the indicated times. TG: thapsigargin, TM: tunicamycin. Both figures show a representative Western blot of BiP induction by TG and TM. Quantification of three repeat TG experiments is shown in the lower panel in Fig. 3A. Results are plotted as mean \pm SEM. Significantly different from control (black bars) baseline: ${ }^{*} \mathrm{p}<0.001$; significantly different from Klotho-treated (gray bars) baseline: \# p<0.01; significantly different from respective control at 6 hours: $\uparrow p<0.001$. TM experiment performed once to confirm TG results. C) A549 cells stably transfected with Klotho were treated with thapsigargin and probed for BiP. This experiment was performed once to document similar results in different cell types. Klotho overexpression in A549 cells was determined by probing the membrane with anti-Flag. All blots were probed with $\beta$-actin for loading control.

effect of Klotho was observed in TM-treated cells as early as 2 hrs. In order to demonstrate the Klotho effect is generalized, A549 cells stably transfected with Klotho were treated with TG for the indicated times, and probed for BiP (Fig. 3C). A protective effect of Klotho is present as early as $4 \mathrm{hrs}$ after initiation of TG exposure.

\section{Loss of Klotho increases susceptibility to ER stress}

Transfection of HEK293 cells with Klotho siRNA resulted in a $60 \%$ decrease in Klotho assessed with RT-PCR (data not shown). $48 \mathrm{hrs}$ post-transfection, cells were treated with TG (500 nM), and phospho-IRE-1, total IRE-1, XBP-1s and BiP levels were assayed using Western blotting. These ER stress markers were increased in Klotho-silenced cells vs cells transfected with control siRNA as early as 2 hrs for phospho-IRE-1 (Fig. 4A), 4 hrs for XBP1, and 4-6 hrs for BiP (Fig. 4B). While loss of Klotho increased phospho-IRE-1 after 2 and 4 hours, these differences were not statistically significant by 2 way ANOVA testing $(\mathrm{p}<0.084)$, primarily due to the decrease in the Klotho group at 6 hours.

\section{Klotho modulates ER stress activated cell death pathways}

UPR-associated signaling events are initially intended to reestablish homeostasis and normal ER function by activating transcriptional factors that induce expression of genes capable of enhancing the protein folding capacity of the ER, and genes for ER-associated degradation (ERAD) to help clear the ER of unfolded proteins and export them for cytosolic degradation. When the UPR adaptive mechanisms fail, cell death is induced typically by apoptosis [36]. Among the major UPR signal transduction pathways, the IRE-1 pathway has been most clearly connected to cell death, as it leads to activation of stress kinases, including 
Fig. 4. Klotho knockdown sensitized cells to ER stress. HEK293 cells transfected with either control scrambled siRNA (C) or Klotho specific siRNA (K) for $48 \mathrm{hrs}$ before treating with thapsigargin (500 $\mathrm{nM}$ ) for indicated times. A representative Western blot demonstrates effect of Klotho knockdown on A) phospho- and total IRE-1 protein levels. Quantification of three repeat TG experiments is shown in the lower panel in Fig. 4A. Results are plotted as mean \pm SEM. Significantly different from control (black bars) baseline: ${ }^{*} \mathrm{p}<0.001$; significantly different from Klotho-treated (gray bars) baseline: \# p<0.05, \#\# p<0.001; significantly different from respective control at 6 hours: $† p<0.01$ B) XBP-1s and BiP protein levels. Quantification of three repeat TG experiments is shown in the two lower panels in Fig. 4B for XBP$1 \mathrm{~s}$ and BiP. Results are plotted as mean \pm SEM. For XBP-1s, significantly different from control (black bars) baseline: ${ }^{*} \mathrm{p}<0.001$; significantly different from Klotho-treated (gray bars) baseline: \# $\mathrm{p}<0.001$; significantly different from respective control at 4 hours: $\dagger p<0.05$. For BiP, significantly different from control (black bars) baseline: * $\mathrm{p}<0.05$, ** $\mathrm{p}<0.001$; significantly different from Klotho-treated (gray bars) baseline: \# p<0.001; significantly different from respective control at 4 and 6 hours: $\uparrow \mathrm{p}<0.01$. $\beta$-actin is shown as loading control.

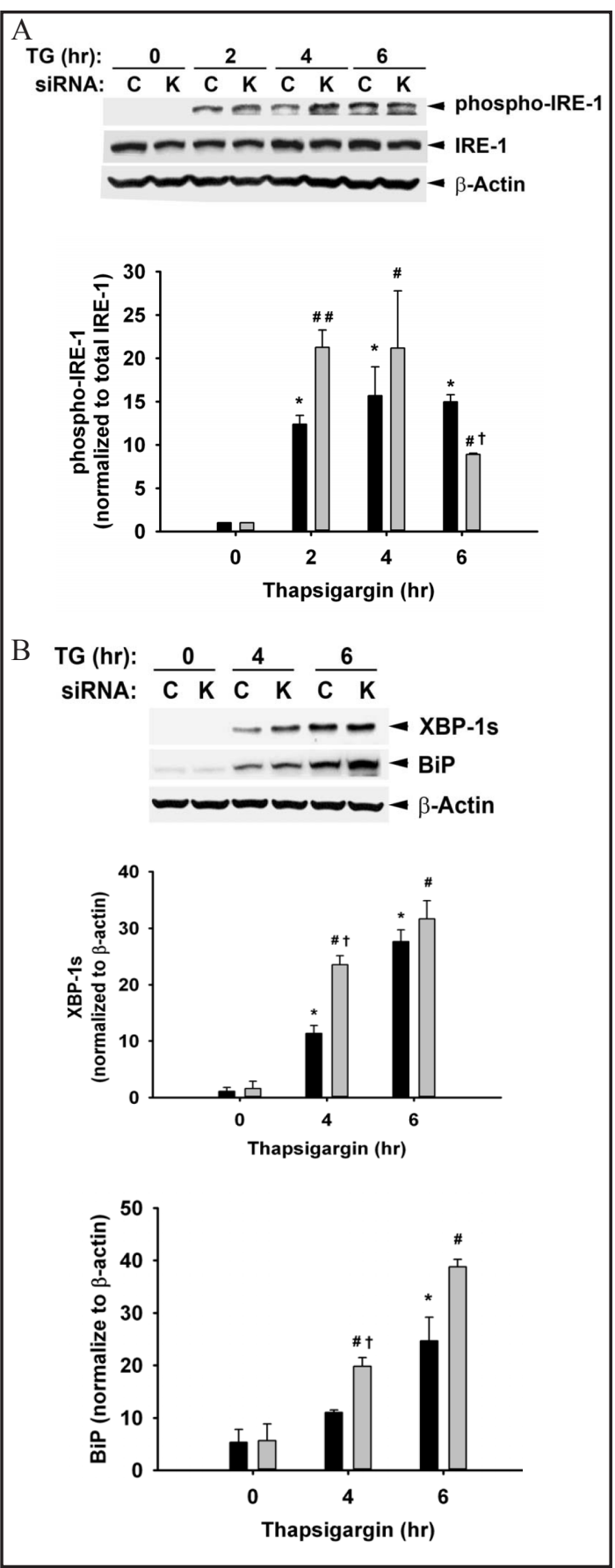

p-38 and JNK [37]. JNK and p38 kinase are proline-directed serine/threonine kinases that are frequently activated after cells are exposed to toxic chemicals and/or environmental stress such as UV exposure, $\gamma$-ray irradiation and agents that trigger ER stress [38].

Since ER stress culminates in the activation of the proapoptotic kinases JNK and p38 [39], we investigated if Klotho modulated JNK and p38 kinase activity downstream of IRE-1 and PERK signaling. Phosphorylation of JNK and p38 kinase, determined by Western blot analysis using specific antibodies to phosphorylated forms of both kinases, increased after TG treatment (Figs. 5A and 5B, respectively). Both JNK and p38 kinase phosphorylation was suppressed in Klotho stable cell line, whereas the levels of non-phosphorylated (inactive) JNK and p38 kinase were comparable in all samples (Figs. 5A and B). Our results suggest 


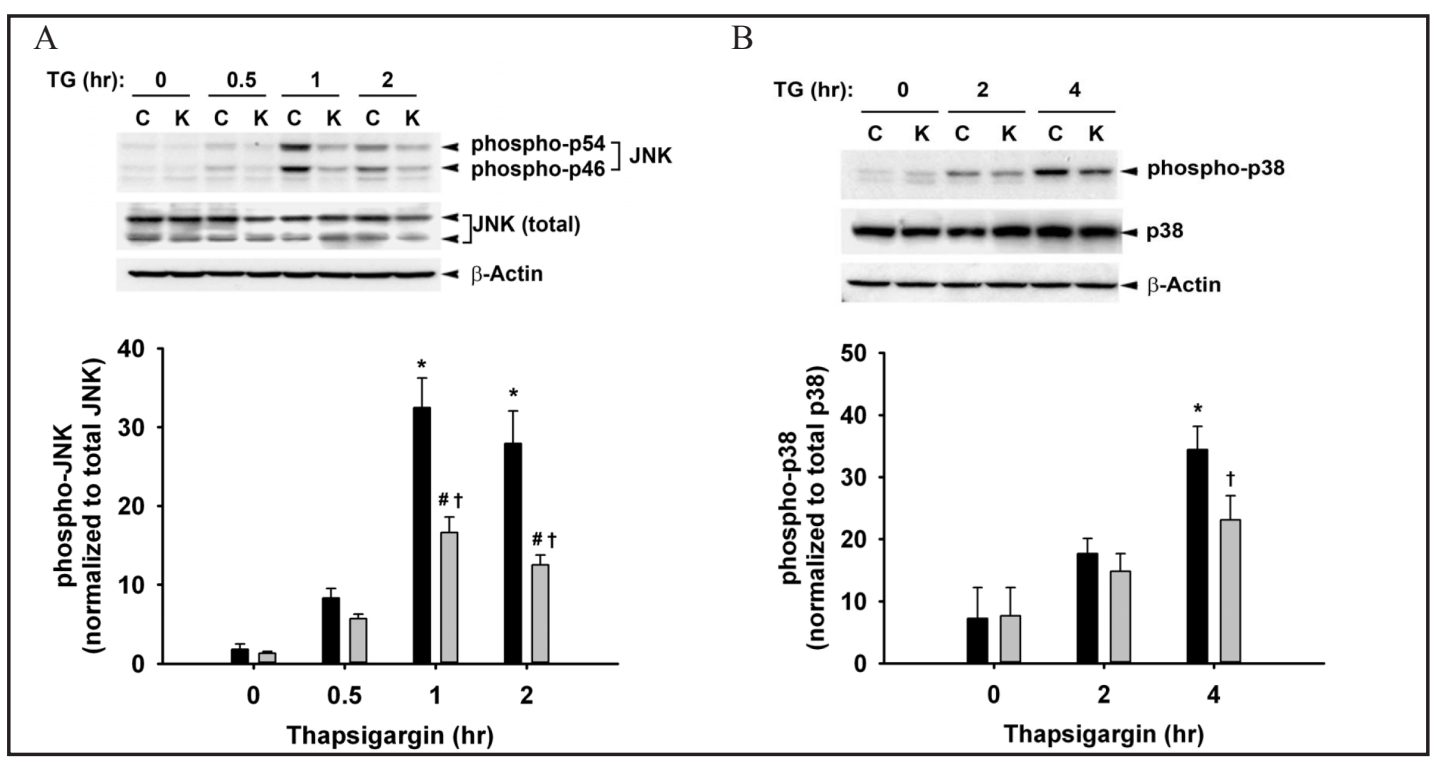

Fig. 5. Klotho expression inhibited thapsigargin-induced JNK and p38 phosphorylation. HK-2 cells expressing Klotho (K) and the control vector (C) were treated with thapsigargin (500 $\mathrm{nM}$ ) for indicated times. Activation of MAPKs was detected with A) anti-phospho-JNK. Quantification of three repeat TG experiments is shown in the lower panel in Fig. 5A. Results are plotted as mean \pm SEM. Significantly different from control (black bars) baseline: * $\mathrm{p}<0.001$; significantly different from Klotho-treated (gray bars) baseline: \# $<<0.05$; significantly different from respective control at 1 and 2 hours: $\dagger p<0.001$. B) phospho-p38. Quantification of two repeat TG experiments is shown in the lower panel in Fig. 5B. Results are plotted as mean \pm SEM. Significantly different from control (black bars) baseline: ${ }^{*} \mathrm{p}<0.01$; significantly different from respective control at 4 hours: $\uparrow \mathrm{p}<0.005$. Total proteins were measured by probing with anti-JNK and p38 antibodies. $\beta$-actin served as a loading control.

that Klotho modulates apoptosis through the IRE1-initiated pathway via inactivation of JNK and p38 stress kinases.

The downstream elements of all three UPR pathways converge on the promoter of the CHOP gene that has been implicated in the cellular response to stress. It is a bZIP family transcription factor that reportedly induces expression of the pro-apoptotic protein Bim and suppresses expression of anti-apoptotic protein Bcl-2 [40]. These events have been causally linked to ER stress-induced apoptosis. In HK-2 cells stably transfected with Klotho, induction of CHOP mRNA expression was $50 \%$ less than the induction in the control cell line (Fig. 6A). A similar effect of Klotho overexpression on TG-induced CHOP was observed at protein levels (Fig. 6B). Consistent results were observed in A549 cells stably transfected with control vector or Klotho, then treated with TG for extended time intervals (Fig. 6C). We observed induction in CHOP 16 hrs after TG treatment that was sustained through $24 \mathrm{hrs}$ but reduced after $48 \mathrm{hrs}$ (Fig. 6C). Klotho overexpressing cells showed a lower level of CHOP induction, especially after 24 and 48 hrs of TG treatment. These results suggest that Klotho expression may protect cells from ER stress-induced cell death.

ER stress-induced cell death is usually mediated via activation of apoptotic signals. Thus, we further investigated the effect of Klotho expression on TG-induced caspase and PARP cleavage. TG treatment caused a time-dependent increase in the cleavage of various caspases involved in ER stress-mediated apoptosis, including caspase-3, -9 and -7 in control cells. Klotho overexpressing stable cells displayed no increase in caspase cleavage through $24 \mathrm{hrs}$ and lower levels of cleaved caspases after $48 \mathrm{hrs}$ of TG exposure versus controls (Fig. 7A). Induction in PARP cleavage was observed in control cells after $48 \mathrm{hrs}$ of TG treatment, with substantially lower levels of cleaved PARP detected in Klotho expressing stable cells (Fig. 7B). Taken together, these data suggest an anti-apoptotic role of Klotho under cell 
Fig. 6. Klotho inhibited CHOP induction in response to thapsigargin. A) Real-time PCR analysis demonstrating mRNA levels of CHOP in control and Klotho-overexpressing HK-2 cells. Data represent mean \pm SD of three replicate experiments. ${ }^{*} \mathrm{p}<0.001$ by Student's t test. B) Representative Western blot indicating CHOP protein levels after thapsigargin treatment in control and Klotho-overexpressing HK-2 cells. C) A549 cells stably transfected with Klotho were treated with thapsigargin and probed for CHOP. Klotho was determined by probing the membrane with anti-Flag. Blots were probed with $\beta$-actin for loading control.

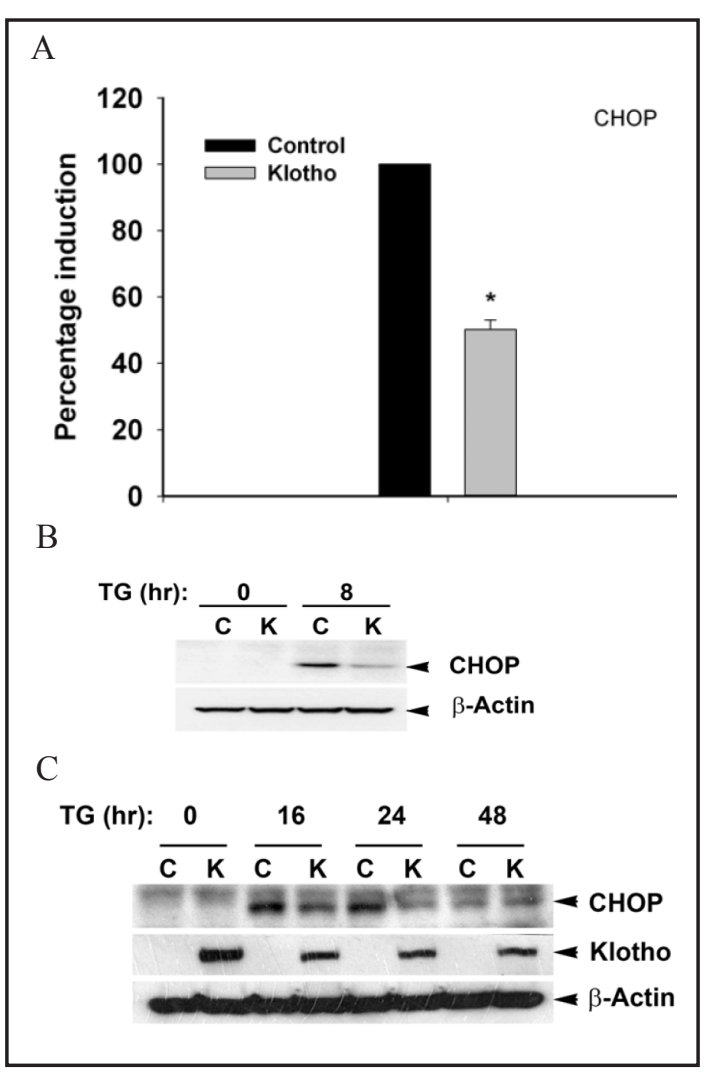

conditions of ER-stress. This was substantiated further by a cell survival assay, where Klotho overexpressing cells demonstrated significantly higher cell survival over a wide range of TG doses compared to control cells (Fig. 8).

\section{Discussion}

The anti-aging function of Klotho has been attributed to its ability to modulate phosphate and 1,25-dihydroxy-vitamin D metabolism, and its function in combating oxidative stress has been linked to its regulation of key enzymes involved in anti-oxidative pathways [41]. Recently, we have reported that Klotho regulates inflammation by suppressing NF$\kappa \mathrm{B}$ activation in response to various inflammatory stimuli by inhibiting RelA serine 536 phosphorylation and RelA transactivation. In a senescence model, anti-inflammatory effects of Klotho are reported to be mediated by its interaction with retinoic acid-inducible gene-I (RIG-I) [42]. Klotho appears to have an important role in modulating cellular stress pathways and thus, loss of Klotho has been associated with increased inflammation and oxidative stress in various diseases and conditions. Since these cellular stresses also have been linked to ER stress, we explored the potential role of Klotho in regulating ER stress, and have shown that increased Klotho expression mitigates ER stress. Conversely, decreases in Klotho activity in an in vitro cell culture model increased the cellular ER stress response.

Our data therefore suggest that Klotho activity may play an important role in alleviating the pathophysiological insults that lead to the accumulation of unfolded proteins in the ER and aberrant activation of ER stress. Our data support the idea that Klotho activity might play an important protective role in mitigating ER stress that arises from hypoxia, nutrient deprivation, alterations in the redox balance, changes in calcium homeostasis, and failure of posttranslational modifications [43]. Evaluation and monitoring of ER stress are required for investigation of molecular events involved in ER stress-related pathophysiologies. Based on the above-mentioned knowledge on UPR, several methods have been used to assess ER 


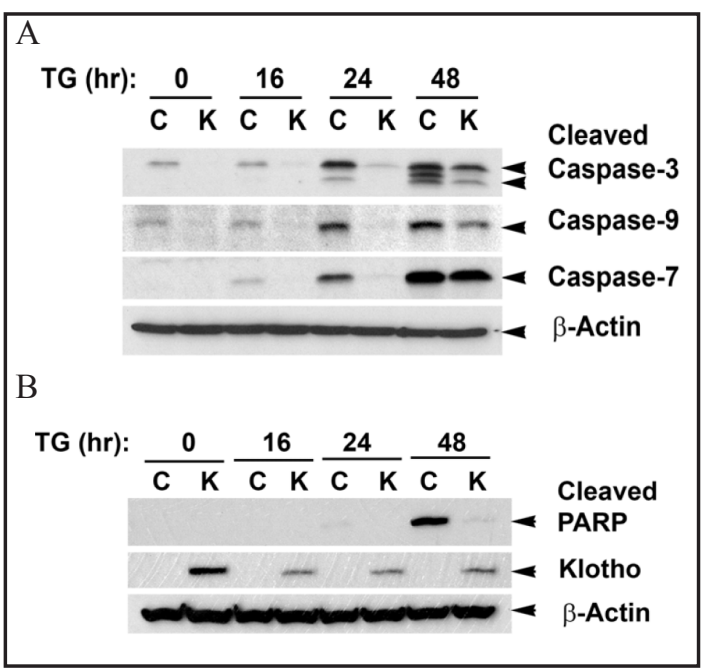

Fig. 7. Klotho inhibits thapsigargin-induced PARP cleavage and caspase activation. A549 cells stably transfected with Klotho $(\mathrm{K})$ or control vector (C) were cultured for 16, 24 and $48 \mathrm{hrs}$ in the presence or absence of thapsigargin (500 $\mathrm{nM})$, and cell lysates were used for immunoblotting to probe for A) cleaved caspase-3, -7 and -9 or B) PARP. Blots probed with $\beta$-actin for loading control. Klotho was determined by probing the membrane with anti-Flag.

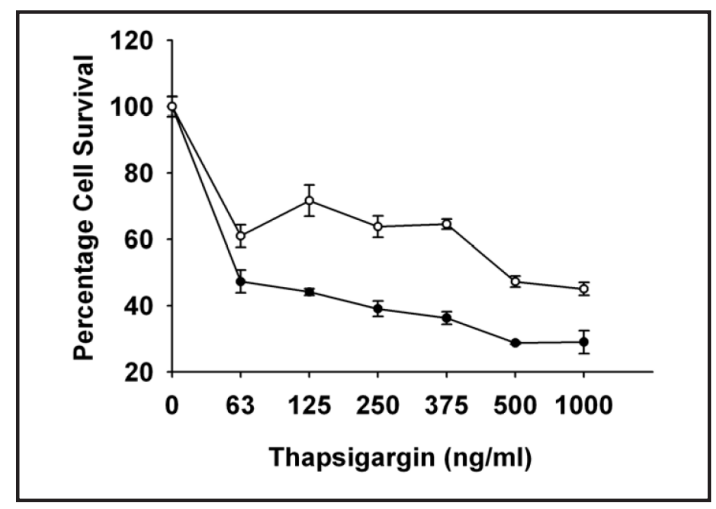

Fig. 8. Klotho prevented thapsigargin-induced cell death. A549 Klotho stable and control cells were treated with thapsigargin at different concentrations for 24 hrs. Cell viability was measured by MTT assay as described in Materials and Methods. Quantification of three repeat TG experiments is shown with the data presented as mean \pm SD. Filled circles, control; open circles, Klotho overexpressing cells. 2 way ANOVA indicated significant differences $(\mathrm{p}<0.03)$, and Tukey's post-hoc test for pairwise differences was significant $(p<0.001)$ for all thapsigargin concentrations.

stress in pathophysiological conditions. Expression of endogenous biomarkers, e.g., GRP78 and CHOP, is most commonly used for this purpose [22, 44]. Our results demonstrate that cells overexpressing Klotho are resistant to chemical-induced ER stress and activation of ER stress sensor BiP/Grp78 compared with control cells. Phosphorylation of IRE-1 $\alpha$, PERK and eIF $2 \alpha$, cleavage of ATF 6 and procaspase- 12 , and splicing of XBP-1 mRNA have also been used as endogenous indicators for ER stress $[45,46]$. Activated PERK phosphorylates eukaryotic translation initiation factor 2 subunit $\alpha$ (eIF $2 \alpha$ ). After stress-induced phosphorylation of eIF2 $\alpha$, global protein translation of normal cellular mRNAs is repressed [1].

Prosurvival or anti-apoptotic role of Klotho has been appreciated in different apoptotic models. A genetically manipulated mouse model of progressive renal injury showed a significant improvement in renal function along with reduction in oxidative stress and apoptosis [47]. In this context, Sugiura et al. reported that Klotho reduced apoptosis in experimental ischemic acute kidney injury by increasing HSP-70 [48]. Similarly, Klotho overexpression attenuated cellular apoptosis and senescence in vascular cells via mitogenactivated kinase kinase and extracellular-regulated kinase pathway after hydrogen peroxide treatment [49]. Our data suggest that Klotho can reduce apoptotic signals and increase cell survival after chemical ER-inducer treatment. Here we have shown that Klotho overexpressing cells have lower levels of CHOP expression and reduced IRE-1, PERK and elF2 $\alpha$ phosphorylation and JNK activation after TG treatment. Although we do not yet have a clear mechanism for the anti-apoptotic effect of Klotho in these cells after exposure to a pharmacologic ER inducer, the process does seem to be very different than the cell survival action HSP72 that depends on its ability to bind with IRE1 $\alpha$ and increase XBP1 cleavage [50]. In addition to inhibition of thapsigargin-induced PERK pathway activation, other potential pro-survival mechanisms of Klotho may include: 1) acting as an anti-oxidative stress agent, and 2) interfering with the TRAF2-ASK1-IRE1 $\alpha$ complex formation. Certainly, the well recognized ability of Klotho to increase anti-oxidative enzymes such as superoxide dismutase [25] may decrease cellular and tissue levels of oxygen-derived free radicals that have been implicated in ER stress [51,52]. 
Activation of the IRE-1 arm of UPR signaling is linked to apoptosis via a variety of mechanisms. Activated IRE-1 is coupled to JNK activation by interacting with TRAF-2 and apoptosis signal-regulating kinase-1 (ASK-1) [53]. A recent study suggests that Klotho overexpression suppresses ASK1 activation [54]. Thus, Klotho expression could alter the formation of this pro-apoptotic complex and thereby leading to JNK inhibition. In addition to JNK activation, binding of activated IRE-1 to TRAF-2 causes the release and subsequent cleavage of procaspase-12 into an active enzyme [55]. Our failure to observe caspase-12 activation in our experiments is consistent with the controversial role of capsase- 12 activation in ER stress [56, 57].

In conclusion, our data indicate that higher levels of Klotho expression can ameliorate chemically-induced ER stress by decreasing levels of UPR signaling markers in stressed cells. Our data further suggest that Klotho serves as a part of cellular defense machinery engaged in providing protection against a wide variety of cellular stresses that include oxidative and inflammatory stresses as well as the cellular response to unfolded proteins. Thus, loss of Klotho as observed in diabetes and aging could make cells more vulnerable to these stresses, leading to increased apoptosis and cell death associated with these conditions.

\section{Abbreviations}

ATF6 (activating transcription factor-6); CHOP (CCAAT/enhancer-binding proteinhomologous protein); eIF2 $\alpha$ (eukaryotic translation initiation factor 2 subunit $\alpha$ ); ERAD (ERassisted degradation); GRP78 (glucose-regulated protein, 78kDa); IRE-1 (inositol requiring enzyme-1); JNK (c-Jun N-terminal kinase); PERK (protein kinase RNA activated-like ER kinase); UPR (unfolded protein response); XBP-1(X-box-binding protein 1).

\section{Acknowledgements}

SB and YZ contributed equally. This work was supported, in part, by NIH grant NIDDKDK079053 (RGT and SC), American Heart Association-0630100N (SC) and John Sealy Foundation Grant (PS).

\section{References}

1 Hirosumi J, Tuncman G, Chang L ,Gorgun CZ, Uysal KT, Maeda K, Karin M, Hotamisligil GS: A central role for JNK in obesity and insulin resistance. Nature 2002;420:333-336.

$\checkmark 2$ Uysal KT, Wiesbrock SM, Marino MW, Hotamisligil GS: Protection from obesity-induced insulin resistance in mice lacking TNF-alpha function. Nature 1997;389:610-614.

3 Kaneto H, Nakatani Y, Kawamori D, Miyatsuka T, Matsuoka TA, Matsuhisa M, Yamasaki Y: Role of oxidative stress, endoplasmic reticulum stress, and c-Jun N-terminal kinase in pancreatic beta-cell dysfunction and insulin resistance. Int J Biochem Cell Biol 2006;38:782-793.

4 Yuan M, Konstantopoulos N, Lee J, Hansen L, Li ZW, Karin M, Shoelson SE: Reversal of obesity- and dietinduced insulin resistance with salicylates or targeted disruption of Ikkbeta. Science 2001;293:1673-1677. Ma Y, Hendershot LM: The unfolding tale of the unfolded protein response. Cell 2001;107:827-830. Kaufman RJ, Scheuner D, Schroder M, Shen X, Lee K, Liu CY, Arnold SM: The unfolded protein response in nutrient sensing and differentiation. Nat Rev Mol Cell Biol 2002;3:411-421.

7 Kharroubi I, Ladriere L, Cardozo AK, Dogusan Z, Cnop M, Eizirik DL: Free fatty acids and cytokines induce pancreatic beta-cell apoptosis by different mechanisms: role of nuclear factor-kappaB and endoplasmic reticulum stress. Endocrinology 2004;145:5087-5096.

-8 Mori K: Tripartite management of unfolded proteins in the endoplasmic reticulum. Cell 2000;101:451-454.

-9 Rutkowski DT, Kaufman RJ: A trip to the ER: coping with stress. Trends Cell Biol 2004;14:20-28.

10 Kim R, Emi M, Tanabe K, Murakami S: Role of the unfolded protein response in cell death. Apoptosis 2006;11:5-13. 


\begin{tabular}{|c|c|c|}
\hline Cellular Phys & Cell Physiol Biochem 2013;31:659-672 & \\
\hline and Biochemistry & $\begin{array}{l}\text { DOI: } 10.1159 / 000350085 \\
\text { Publisned onIIne: May U8, } 2013\end{array}$ & $\begin{array}{l}\text { O } 2013 \text { S. Karger AG, Basel } \\
\text { www.karger.com/cpb }\end{array}$ \\
\hline
\end{tabular}

11 Harding HP, Zhang Y, Ron D: Protein translation and folding are coupled by an endoplasmic-reticulumresident kinase. Nature 1999;397:271-274.

12 Bertolotti A, Zhang Y, Hendershot LM, Harding HP, Ron D: Dynamic interaction of BiP and ER stress transducers in the unfolded-protein response. Nat Cell Biol 2000;2:326-332.

$\checkmark 13$ Liu CY, Schroder M, Kaufman RJ: Ligand-independent dimerization activates the stress response kinases IRE1 and PERK in the lumen of the endoplasmic reticulum. J Biol Chem 2000;275:24881-24885.

14 Okada T, Haze K, Nadanaka S, Yoshida H, Seidah NG, Hirano Y, Sato R, Negishi M, Mori K: A serine protease inhibitor prevents endoplasmic reticulum stress-induced cleavage but not transport of the membranebound transcription factor ATF6. J Biol Chem 2003;278:31024-31032.

15 Yoshida H, Matsui T, Yamamoto A, Okada T, Mori K: XBP1 mRNA is induced by ATF6 and spliced by IRE1 in response to ER stress to produce a highly active transcription factor. Cell 2001;107:881-891.

-16 Calfon M, Zeng H, Urano F, Till JH, Hubbard SR, Harding HP, Clark SG, Ron D: IRE1 couples endoplasmic reticulum load to secretory capacity by processing the XBP-1 mRNA. Nature 2002;415:92-96.

17 Harding HP, Zhang Y, Bertolotti A, Zeng H, Ron D: Perk is essential for translational regulation and cell survival during the unfolded protein response. Mol Cell 2000;5:897-904.

18 Dorner AJ, Wasley LC, Kaufman RJ: Overexpression of GRP78 mitigates stress induction of glucose regulated proteins and blocks secretion of selective proteins in Chinese hamster ovary cells. EMBO J 1992;11:1563-1571.

19 Leborgne-Castel N, Jelitto-Van Dooren EP, Crofts AJ, Denecke J: Overexpression of BiP in tobacco alleviates endoplasmic reticulum stress. Plant Cell 1999;11:459-470.

20 Little E, Lee AS: Generation of a mammalian cell line deficient in glucose-regulated protein stress induction through targeted ribozyme driven by a stress-inducible promoter. J Biol Chem 1995;270:9526-9534.

21 Morris JA, Dorner AJ, Edwards CA, Hendershot LM, Kaufman RJ: Immunoglobulin binding protein (BiP) function is required to protect cells from endoplasmic reticulum stress but is not required for the secretion of selective proteins. J Biol Chem 1997;272:4327-4334.

22 Ma Y, Brewer JW, Diehl JA, Hendershot LM: Two distinct stress signaling pathways converge upon the CHOP promoter during the mammalian unfolded protein response. J Mol Biol 2002;318:1351-1365.

-23 Kuro-o M, Matsumura Y, Aizawa H, Kawaguchi H, Suga T, Utsugi T, Ohyama Y, Kurabayashi M, Kaname T, Kume E, Iwasaki H, Iida A, Shiraki-Iida T, Nishikawa S, Nagai R, Nabeshima YI: Mutation of the mouse klotho gene leads to a syndrome resembling ageing. Nature 1997;390:45-51.

24 Kurosu H, Yamamoto M, Clark JD, Pastor JV, Nandi A, Gurnani P, McGuinness OP, Chikuda H, Yamaguchi M, Kawaguchi H, Shimomura I, Takayama Y, Herz J, Kahn CR, Rosenblatt KP, Kuro-o M: Suppression of aging in mice by the hormone Klotho. Science 2005;309:1829-1833.

25 Yamamoto M, Clark JD, Pastor JV, Gurnani P, Nandi A, Kurosu H, Miyoshi M, Ogawa Y, Castrillon DH, Rosenblatt KP, Kuro-o M: Regulation of oxidative stress by the anti-aging hormone klotho. J Biol Chem 2005;280:38029-38034.

-26 Wang Y, Sun Z: Current understanding of klotho. Ageing Res Rev 2009;8:43-51.

27 Kops GJ, Dansen TB, Polderman PE, Saarloos I, Wirtz KW, Coffer PJ, Huang TT, Bos JL, Medema RH, Burgering BM: Forkhead transcription factor FOXO3a protects quiescent cells from oxidative stress. Nature 2002;419:316-321.

28 Kuro-o M: Klotho as a regulator of oxidative stress and senescence. Biol Chem 2008;389:233-241.

29 Ikushima M, Rakugi H, Ishikawa K, Maekawa Y, Yamamoto K, Ohta J, Chihara Y, Kida I, Ogihara T: Antiapoptotic and anti-senescence effects of Klotho on vascular endothelial cells. Biochem Biophys Res Commun 2006;339:827-832.

-30 Zhao Y, Banerjee S, Dey N, LeJeune WS, Sarkar PS, Brobey R, Rosenblatt KP, Tilton RG, Choudhary S: Klotho depletion contributes to increased inflammation in kidney of the $\mathrm{db} / \mathrm{db}$ mouse model of diabetes via RelA (serine)536 phosphorylation. Diabetes 2011;60:1907-1916.

-31 Livak KJ, Schmittgen TD: Analysis of relative gene expression data using real-time quantitative PCR and the 2(-Delta Delta C(T)) Method. Methods 2001;25:402-408.

-32 Shamu CE, Walter P: Oligomerization and phosphorylation of the Ire1p kinase during intracellular signaling from the endoplasmic reticulum to the nucleus. EMBO J 1996;15:3028-3039.

-33 Welihinda AA, Kaufman RJ: The Unfolded Protein Response Pathway in Saccharomyces cerevisiae. J Biol Chem 1996;271:18181-18187.

-34 Lee AH, Iwakoshi NN, Glimcher LH: XBP-1 regulates a subset of endoplasmic reticulum resident chaperone genes in the unfolded protein response. Mol Cell Biol 2003;23:7448-7459. 
35 Chen G, Ma C, Bower KA, Shi X, Ke Z, Luo J: Ethanol promotes endoplasmic reticulum stress-induced neuronal death: Involvement of oxidative stress. J Neurosci Res 2008;86:937-946.

-36 Kim I, Xu W, Reed JC: Cell death and endoplasmic reticulum stress: disease relevance and therapeutic opportunities. Nat Rev Drug Discov 2008;7:1013-1030.

-37 Tobiume K, Matsuzawa A, Takahashi T, Nishitoh H, Morita K, Takeda K, Minowa O, Miyazono K, Noda T, Ichijo H: ASK1 is required for sustained activations of JNK/p38 MAP kinases and apoptosis. EMBO Rep 2001;2:222-228.

38 Xu C, Bailly-Maitre B, Reed JC: Endoplasmic reticulum stress: cell life and death decisions. J Clin Invest 2005;115:2656-2664.

-39 Kim I, Shu CW, Xu W, Shiau CW, Grant D, Vasile S, Cosford ND, Reed JC: Chemical biology investigation of cell death pathways activated by endoplasmic reticulum stress reveals cytoprotective modulators of ASK1. J Biol Chem 2009;284:1593-1603.

40 McCullough KD, Martindale JL, Klotz LO, Aw TY, Holbrook NJ: Gadd153 sensitizes cells to endoplasmic reticulum stress by down-regulating Bcl2 and perturbing the cellular redox state. Mol Cell Biol 2001;21:1249-1259.

41 Kuro-o M: A potential link between phosphate and aging - Lessons from Klotho-deficient mice. Mech Ageing Dev 2010;131:270-275.

-42 Liu F, Wu S, Ren H, Gu J: Klotho suppresses RIG-I-mediated senescence-associated inflammation. Nat Cell Biol 2011;13:254-262.

43 Lee AS: The ER chaperone and signaling regulator GRP78/BiP as a monitor of endoplasmic reticulum stress. Methods 2005;35:373-381.

44 Kozutsumi Y, Segal M, Normington K, Gething MJ, Sambrook J: The presence of malfolded proteins in the endoplasmic reticulum signals the induction of glucose-regulated proteins. Nature 1988;332:462-464.

45 Nakagawa T, Zhu H, Morishima N, Li E, Xu J, Yankner BA, Yuan J: Caspase-12 mediates endoplasmicreticulum-specific apoptosis and cytotoxicity by amyloid-[beta]. Nature 2000;403:98-103.

-46 Schroder M, Kaufman RJ: ER stress and the unfolded protein response. Mutation Research/Fundamental and Molecular Mechanisms of Mutagenesis 2005;569:29-63.

47 Haruna Y, Kashihara N, Satoh M, Tomita N, Namikoshi T, Sasaki T, Fujimori T, Xie P, Kanwar YS: Amelioration of progressive renal injury by genetic manipulation of Klotho gene. Proc Natl Acad Sci USA 2007;104:23312336.

48 Sugiura H, Yoshida T, Mitobe M, Yoshida S, Shiohira S, Nitta K, Tsuchiya K: Klotho reduces apoptosis in experimental ischaemic acute kidney injury via HSP-70. Nephrol Dial Transplant 2010;25:60-68.

49 Maekawa Y, Ohishi M, Ikushima M , Yamamoto K, Yasuda O, Oguro R, Yamamoto-Hanasaki H, Tatara Y, Takeya Y, Rakugi H: Klotho protein diminishes endothelial apoptosis and senescence via a mitogenactivated kinase pathway. Geriatr Gerontol Int 2011;11:510-516.

-50 Gupta S, Deepti A, Deegan S, Lisbona F, Hetz C, Samali A: HSP72 Protects Cells from ER Stress-induced Apoptosis via Enhancement of IRE1 $\alpha$-XBP1 Signaling through a Physical Interaction. PLoS Biol 2010;8:e1000410.

-51 Higa A, Chevet E: Redox signaling loops in the unfolded protein response. Cell Signal 2012;24:1548-1555.

52 Wang S, Kaufman RJ: The impact of the unfolded protein response on human disease. J Cell Biol 2012;197:857-867.

53 Nishitoh H, Matsuzawa A, Tobiume K, Saegusa K, Takeda K, Inoue K, Hori S, Kakizuka A, Ichijo H: ASK1 is essential for endoplasmic reticulum stress-induced neuronal cell death triggered by expanded polyglutamine repeats. Genes Dev 2002;16:1345-1355.

54 Hsieh CC, Kuro-o M, Rosenblatt KP, Brobey R, Papaconstantinou J: The ASK1-Signalosome regulates p38 MAPK activity in response to levels of endogenous oxidative stress in the Klotho mouse models of aging. Aging (Albany NY) 2010;2:597-611.

55 Yoneda T, Imaizumi K, Oono K, Yui D, Gomi F, Katayama T, Tohyama M: Activation of caspase-12, an endoplastic reticulum (ER) resident caspase, through tumor necrosis factor receptor-associated factor 2-dependent mechanism in response to the ER stress. J Biol Chem 2001;276:13935-13940.

-56 Saleh M, Mathison JC, Wolinski MK, Bensinger SJ, Fitzgerald P, Droin N, Ulevitch RJ, Green DR, Nicholson DW: Enhanced bacterial clearance and sepsis resistance in caspase-12-deficient mice. Nature 2006;440:1064-1068.

57 Xue Y, Daly A, Yngvadottir B Liu M, Coop G, Kim Y, Sabeti P, Chen Y, Stalker J, Huckle E, Burton J, Leonard S, Rogers J, Tyler-Smith C: Spread of an inactive form of caspase-12 in humans is due to recent positive selection. Am J Hum Genet 2006;78:659-670. 